International Journal of Wireless \& Mobile Networks (IJWMN) Vol. 5, No. 6, December 2013

\title{
Novel Cell Selection Procedure for LTE HETNETS BASED ON MATHEMATICAL MODELLING of Proportional Fair Scheduling
}

\author{
Mohamed A. AboulHassan ${ }^{1}$, Essam A. Sourour ${ }^{2}$, Shawki Shaaban ${ }^{2}$ \\ ${ }^{1}$ Pharos University, Faculty of Engineering, Electrical Eng. Dept., Alexandria, Egypt \\ ${ }^{2}$ Alexandria University, Department of Electrical Eng., Alexandria, 21544 ,Egypt
}

\begin{abstract}
Femtocells have been considered one of the most important technologies in LTE networks to solve indoor coverage problem, however the randomness deployment of femtocells, leads to great challenge for selecting optimum serving cell. In this work, a new cell selection algorithm is proposed that enables new user to select best serving cell whereas several factors are put into consideration other than highest instantaneous SNR or maximum RSRP such as cell load.A new prediction algorithm is designed to predict the performance of $(P F)$ scheduling algorithm to calculate expected number of RBs to be scheduled to new user, then reduction in achievable data rate due to both received SNR and instant cell load is estimated. The numerical results show that the new proposed cell selection algorithm achieves higher average cell throughput than conventional cell selection methods and achieves less cell load variance between different adjacent cells.
\end{abstract}

\section{KEYWORDS}

LTE; Proportional Fair scheduling algorithm, cell selection

\section{INTRODUCTION}

LTE (Long Term Evolution) [1] is a standard for wireless communication of high-speed data for mobile phones; it aims to achieve higher data rates meeting the rapid growth in demanded mobile applications. As, the percentage of traffic indoors has been increasing over time and is expected to increase further, good indoor coverage has been considered nowadays as one of the main design aspects to achieve target peak data rate, therefore low power nodes such as femtocells has been introduced as an effective solution. However, the existence of different network elements having distinct maximum transmit power that leads to the necessity of more complicated algorithm to achieve target peak data rate, Moreover, due to the shared technology of downlink shared channel in LTE networks, cell load participates in the parameters of the user's achievable data rate. Various Load balancing algorithms [2-4] have been proposed to maintain more balanced load for better average cell throughput. Other researches focus on improving the Resource Allocation (RA) procedure. Cell selection is considered one of important key points whereas optimum serving cell selection algorithms saves necessary downlink RA procedures, and load balancing operations. Conventional cell selection methods depending on highest instantaneous signal-to-noise ratio (SNR) or maximum reference signal received power (RSRP) have been studied to improve cell selection procedure, however such conventional schemes become inefficient due to the dependence of user's achievable data rate on cell load because of the shared resources technology implemented in the main LTE traffic channel (DPSCH), and the difference in transmitted power levels of LTE Heterogeneous Networks (HetNets) elements such as macrocells and femtocells. This may lead to degradation in user's average data throughput and DOI : 10.5121/ijwmn.2013.5603 
consequently needs more dynamic cell selection algorithms. Several researches such as in [5-8] focus on improving conventional systems depending on maximizing instantaneous SNR or reference signal received power (RSRP) whereas in [9], the cell selection algorithms in heterogeneous networks are studied by considering the effect of different maximum transmitted power for different types of base stations. In [10] the effect of implementing Range expansion (RE) [11] is addressed. In order to get higher user's average throughput, cell selection algorithms have considered several factors other than received signal power level, as in [12] and [13], a long term analysis is investigated by considering the relation between cell load and instantaneous received signal level which is used by new user to select the best serving cell, such long term analysis is modelled as an optimization problem which needs a certain level of complexity that might not meet low latency targets.

In this paper, a novel cell selection algorithm is proposed by deriving a mathematical modelling of Proportional Fair (PF) scheduling algorithm [14] which is considered as one of the main scheduling algorithms used in LTE, the proposed algorithm can be applied for both short term and long term analysis, and doesn't need a complex operations as in algorithms based on optimization problems. First we design an algorithm to predict the behaviour of PF scheduling algorithm in future without a necessity to run the PF algorithm. The new prediction algorithm is then used by the new proposed cell selection algorithm to enable new coming user to select best serving cell with minimum expected reduction in achievable user's average data rate due to scheduling within existing users. We also design a standalone new proposed algorithm without a need for an extra overhead due to information exchange between different surrounding cells.

The rest paper is organized as follows; in section II new algorithm for modelling PF scheduling algorithm is proposed. Section III describes the new cell selection algorithm depending on PF Prediction algorithm presented in section II. Simulation results are shown in section IV. Finally section $\mathrm{V}$ yields concluding remarks.

\section{NEW PROPOSED PREDICTION ALGORITHM}

\subsection{PF scheduling Algorithm}

LTE mobile networks rely on scheduling available resources among existing users to achieve an acceptable compromise between fairness and individual user's throughput, therefore the base station needs to run appropriate algorithms to take decisions periodically for assignment of available resources. The minimum resource unit can be assigned to a single user is called Resource Block (RB). One of the most commonly used scheduling algorithms is Proportional fair (PF) algorithm. Previous research as in [15] and [16] has shown that PF scheduling algorithm achieves good balance between user's fairness and average cell throughput, therefore PF algorithm is considered as a standard scheduling algorithm in LTE networks. The work in [17] and [18] has focused on finding a closed form for the user's throughput and system throughput, nevertheless we still need to predict the pattern of served users implementing PF algorithm

The PF depends on selecting the user algorithm having the metric $\mathrm{M}$ defined as

$$
M=\arg \max _{i}\left(\frac{\mathrm{r}_{\mathrm{i}}}{\mathrm{R}_{\mathrm{i}}}\right)
$$

And 
International Journal of Wireless \& Mobile Networks (IJWMN) Vol. 5, No. 6, December 2013

$$
R_{i}(t+1)=\left\{\begin{array}{cc}
R_{i}(t) *\left(1-\frac{1}{t_{c}}\right) & \text { user } i \text { is scheduled } \\
R_{i}(t) *\left(1-\frac{1}{t_{c}}\right)+r_{i}(t) * \frac{1}{t_{c}} & \text { user } i \text { is not scheduled }
\end{array}\right.
$$

Where $\operatorname{Ri}(t)$ is the average moving throughput of user $i$ over a time window $\left(t_{c}\right)$ of an appropriate size, $R_{i}(t+1)$ is updated value after every assigned RB. $r_{\mathrm{i}}(t)$ is the achievable data rate of user $i$ and can be calculated as follows [19]:

$$
r_{i}(t)=\frac{\text { nbits }_{i, j}(t)}{\text { symbol }} * \frac{n s y m b o l s}{\text { slot }} * \frac{n s l o t s}{T T I} * \frac{n s c}{R B}
$$

Values of $r_{i}(\mathrm{t})$ can be obtained from mapping the values of instantaneous Signal-to-Noise Ratio (SNR) into values of achievable Data rate as in table 1 [20]

SNR is given by:

$$
S N R=\frac{P_{t}}{R b n o} \times \frac{10^{-\frac{S H L}{10}}}{(I+N)}
$$

Where $P_{t}$ is the maximum transmitted power value, Rbno is number of RBs per TTI, PL is the path loss value ,SHL is Shadowing fading, $N$ is the white noise power and $I$ is the inter-cell interference from other cells. In the following subsection, we will propose the new prediction algorithm that estimates the pattern of scheduled users i.e. the ID of scheduled users within each $\mathrm{RB}$ without the need to run the PF algorithm and updating vales of average moving throughput $\mathrm{R}$ for each user after each RB

Table 1. values of SNR and their corresponding achievable data rate

\begin{tabular}{|c|c|c|}
\hline $\begin{array}{c}\text { Minimum Instantaneous } \\
\text { Downlink SNR Value }(\mathrm{dB})\end{array}$ & MCS & $\begin{array}{c}\text { Data } \\
\text { Rate } \\
(\mathrm{kbps})\end{array}$ \\
\hline 1.7 & $\operatorname{QPSK}(1 / 2)$ & 168 \\
\hline 3.7 & $\operatorname{QPSK}(2 / 3)$ & 224 \\
\hline 4.5 & $\operatorname{QPSK}(3 / 4)$ & 252 \\
\hline 7.2 & $16 \mathrm{QAM}(1 / 2)$ & 336 \\
\hline 9.5 & $16 \mathrm{QAM}(2 / 3)$ & 448 \\
\hline 10.7 & 16QAM(3/4) & 504 \\
\hline 14.8 & 64QAM(2/3) & 672 \\
\hline 16.1 & 64QAM(3/4) & 756 \\
\hline
\end{tabular}

\subsection{System model}

Assume a base station (BS) consisting of $U$ active users, each user has initial average moving throughput $R_{i}$ and achievable data rate $r_{i}$ where $i=\{1,2 . . U\}$, the ID of new user is $U+1$.

The user's scheduling rate $S_{i}$ of user $i$ is defined as

$$
S_{i}=\frac{r_{i}}{R_{i}}
$$

It can be concluded that under the assumption of flat fading channel and low mobility environment, the value of instantaneous SNR in (4) depends mainly on path loss value which remains almost constant for a certain interval because the log normal shadowing has zero mean, 
so without loss of generality SNR values assumed to be constant through a certain prediction interval, moreover other assumptions as in [18] considered an average values for measured SNR for long term analysis.

The prediction interval can be divided into two periods:

i) Period(1): considered as transient period where only some of active users are scheduled while other users are not scheduled.

ii) Period(2) : considered as steady state period where all active users are scheduled to be served periodically i.e. performance of PF algorithm tends to be the same as Round Robin (RR) scheme

Due to the shared downlink technology of downlink shared LTE main traffic channel, the achievable Downlink Data rate depends mainly on cell load and scheduling criteria, thus we need to estimate the number of RBs assigned to the new user to find the effective achievable Data rate i.e. Practical data rate of new user with certain achievable data rate after being scheduled within a certain cell. To calculate the number of RBs expected to be assigned to new user, we have to calculate the number of RBs assigned to a new user within transient period and number of RBs assigned in steady state period as follows

$$
n_{u+1}^{R B}=n_{P 1}^{R B}+\frac{\left(n_{t o t a l}^{R B}-\sum_{l=1}^{k} m_{k}\right)}{U+1}
$$

Where $n_{p l}{ }^{R B}$ is number of RBs assigned to new user in transient period, $\sum_{l=1}^{k} m_{k}$ is total number of RBs to be scheduled in transient period and $n_{\text {total }} R B$ is the total number of RBs to be scheduled during prediction interval and equals to

$$
n_{\text {total }}^{R B}=n_{T T I}^{R B} * N_{s f} * N_{f r}
$$

Where $n_{T T I}^{R B}$ is number of RBs per TTI, $N_{s f}$ is number of subframes/frame and $N_{f r}$ is number of frames within prediction interval. The second term in equation (6) represents number of RBs assigned in the steady state period, assuming new user to be served in Round Robin (RR) pattern.

Let $r_{U+1}$ is the new coming user's achievable data rate and $R_{U+1}$ is the new user's average moving throughput ,assumed to be minimum as new user has no history. To get the number of RBs in transient period, we need to predict the new serving pattern of all users i.e. ID of user served in each RB during prediction interval after new user is scheduled. The transient period can be divided into number of phases where each phase $k$ contains $m_{k}$ users, sorted in descending order according to scheduling ratio $S$ where

$$
\begin{gathered}
m_{k}=\left\{m_{k} \leq U \mid S_{i}^{d}>S_{j}^{g} \text { for all } i \text { and } j \leq m_{k}, d \text { and } g \text { are the order of } S \text { in phase } k,\right. \\
\text { and } d<g\}
\end{gathered}
$$

The end user of each phase $k$ is defined as: the user having minimum $S$ and has been served in previous phase $k$-1.In classical PF algorithm, the value of moving average throughput $\mathrm{R}$ for each user is updated after each scheduling decision is taken i.e. after each RB, which will consumes a lot of time and calculations, such massive calculations are avoided in the new prediction algorithm by two main methods:

1- The average moving throughput value for each user is updated after the end of each phase instead of each RB by using equation (9) 


$$
R_{u}(l)=R_{u}(0) f^{N}+r_{u}(1-f)\left(\sum_{k=1}^{N} I_{u k} f^{N-k}\right)
$$

Where

$l:$ current phase ID

$N$ : total number of RBs shceduled in previous phases

$f$ : constant factor equals $\left(1-\frac{1}{t_{c}}\right)$, where $f<1$ i.e. $t_{c}>1$

$I_{u k}$ : elements of serving pattern matrix (SPM) having binary value i.e.

$$
I_{u k}= \begin{cases}1 & \text { if } \text { user } u \text { served in } R B k \\ 0 & \text { otherwise }\end{cases}
$$

SPM is a $m_{S P M} x n_{S P M}$ matrix whose entries are binary value indicating whether user is scheduled or not , $m_{S P M}=U+1$ and $n_{S P M}=n_{\text {total }} R B$.

$$
S P M_{m_{S P M} n_{S P M}}=\left[I_{u k}\right]
$$

where each column in SPM has only one value $=[1]$ and the remaining values $=[0]$ because only one user is scheduled in each RB.

2- The user pattern is predicted only in the transient interval while in steady state period, the number of RBs expected to be assigned to new user is calculated by dividing number of RBs in steady state period by number of users due to the fact that the performance of PF algorithm is similar to Round Robin scheme as it will be proven later..

\subsection{Mathematical modelling of prediction algorithm}

Assume active user has initial value $R_{i}(0)$, and new user has initial value $R_{u+1}(0)$ assumed to be minimum where $R_{u+1}(0) \leq R_{i}(0)$ for all $i=1 \ldots U$ The following theorems are stated to verify the procedure of the proposed prediction algorithm

\subsubsection{Theorem 1:}

This theorem states that Phase 1 always contains one user which is new coming user

Proof

New coming user has no history regardless its achievable data rate ,therefore $R_{U+1}$ has minimum value that leads to maximization of $S_{U+1}$, therefore

$$
S_{U+1}(1) \geq S_{j}(1) \quad \forall j=1 \ldots . . U
$$

where $S_{U+1}(1)$ is the scheduling rate of user $u+1$ in phase 1.

\subsubsection{Theorem 2:}

This theorem states that new user $U+1$ is always the last member in every scheduling set of every phase 
By definition, the members of each phase are determined by users having updated values of $S_{j}>$ $S_{u+1}$ and as stated in theorem 3 the order of users in each phase is not changed, therefore new user is the last user in each phase

\subsubsection{Theorem 3 :}

This theorem states that for any two users $i$ and $j$ having $S_{i}$ and $S_{j}$ respectively where $S_{i}(k)>S_{j}(k)$ in phase $k$, then $S_{i}(k+1)>S_{j}(k+1)$ for all values of $i, j$ and $k$

\section{Proof}

N.B. without loss of generality we will apply the proof on transition from phase 2 to phase 3(phase 1 contains only one user as per theorem 1) to trace the behaviour of users then we will show that it can be generalized on all phases

Consider phase 2 has $m_{2}$ users, $u$ ser $r_{j}$ is the first member in phase two i.e. highest value $S_{j}^{\left(m_{2}\right)}(2)$ after end of phase two, user $U+1$ is the last member in phase two having $S_{U+1}^{\left(m_{2}\right)}(2)$, at end of phase 2 the values of $S$ are

$$
\begin{aligned}
S_{j}^{\left(m_{2}\right)}(2) & =\frac{S_{j}}{f^{m_{2}+1}+S_{j}(1-f)\left(f^{m_{2}-1}\right)} \\
S_{U+1}^{\left(m_{2}\right)}(2) & =\frac{S_{U+1}}{f^{m_{2}+1}+S_{U+1}(1-f)\left(1+f^{m_{2}}\right)}
\end{aligned}
$$

and

$$
S_{j}^{\left(m_{2}\right)}(2)>S_{U+1}^{\left(m_{2}\right)}(2)
$$

then

$$
\frac{S_{j}}{f^{m_{2}+1}+S_{j}(1-f)\left(f^{m_{2}-1}\right)}>\frac{S_{U+1}}{f^{m_{2}+1}+S_{U+1}(1-f)\left(1+f^{m_{2}}\right)}
$$

We assume that $S_{j}$ is the initial value of scheduling rate of users, the superscript indicates the number of RBs in each phase after which $S$ is updated, and we omit the phase ID for simplicity.

To prove theorem 3, we have to prove that the inequality $S_{j}^{(1)}(3)<S_{U+1}^{(1)}(3)$ is impossible, where the inequality indicates values of $S$ after assigning first RB in phase 3 to $u s e r_{j}$

$$
\begin{gathered}
\frac{S_{j}}{f^{m_{2}+2}+S_{j}(1-f)\left(1+f^{m_{2}}\right)}<\frac{S_{U+1}}{f^{m_{2}+2}+S_{U+1}(1-f)\left(f+f^{m_{2}+1}\right)} \\
\therefore S_{U+1} S_{j}(1-f)^{2}\left(1+f^{m_{2}}\right)<\left(S_{U+1}-S_{j}\right) f^{m_{2}+2}
\end{gathered}
$$

Which is impossible because from (11) RHS $<0$ and LHS $>0$ by definition

We can notice that for any two phases $k$ and $k+1$, there will be value $m_{k+1}$ in the power term of LHS, using binomial expansion, this value doesn't lead to change sign of LHS, Therefore we can generalize rule three for any phase $k$ 


\subsubsection{Theorem 4:}

This theorem is considered as more generalization for theorem 3, it states that the order of served $m_{k}$ users in phase $k$ is preserved during all incoming phases

Proof

At end of phase 1

$$
S_{j}^{(1)}(1)>S_{U+1}^{(1)}(1)
$$

where the probability of one user at least will have updated $S$ value greater that the update $S$ value of new user is high due do the range of values of $r$.

then

$$
\begin{aligned}
& \frac{S_{j}}{f}>\frac{S_{U+1}}{f+S_{U+1}(1-f)} \\
& \therefore\left(S_{U+1}-S_{j}\right) f<S_{j} S_{U+1}(1-f)
\end{aligned}
$$

Then after $u s e r j$ is served in phase two and $u \operatorname{ser}_{U+1}$ is in last RB in phase two. To prove theorem 4 , we will show that the inequality in (21) is impossible,

At the end of phase 2 if the order is changed

$$
\begin{aligned}
& \therefore S_{j}^{\left(m_{2}\right)}(2)<S_{U+1}^{\left(m_{2}\right)}(2) \\
& \therefore \frac{S_{j}}{f^{m_{2}+1}+S_{j}(1-f) f^{m_{2}-1}}<\frac{S_{U+1}}{f^{m_{2}+1}+S_{U+1}(1-f)\left(1+f^{m_{2}}\right)} \\
& \therefore\left(S_{U+1}-S_{j}\right) f>S_{j} S_{U+1}(1-f) \frac{\left(1+f^{m_{-}}{ }^{m-1}\right)}{f^{m}}
\end{aligned}
$$

it is obvious that using binomial expansion, the $\frac{\left(1+f^{m}-f^{m-1}\right)}{f^{m}}>1$ for all values of $m$, therefore from (23), equation (21) is impossible. Therefore the order is preserved regardless the length of the phase i.e. for any values $m_{k}$, the order is preserved

\subsubsection{Theorem 5:}

This theorem states that, based on theorems $1,2,3$ and 4, for phase $k$ having $m_{k}$ members equals $U+1$, all users are served in periodic pattern i.e. Round Robin (RR) in steady state period with period length $U+1$ and user $U+1$ is the last member of the period. Under assumption of flat fading channel and low mobility environment, users are then served in a Round Robin (RR) pattern until the end of the prediction interval which can be considered steady state period so no need to run any simulation to calculate the number of RBs assigned to new user for the rest of the prediction interval. This result complies with the results in [18] which proved by simulation that the values of $R$ tend to be constant after certain time.

In LTE networks, users are served through Downlink Physical Shared CHannel (DPSCH) where RBs are assigned to users according to scheduling algorithms. Therefore the average user rate depends not only on instantaneous SNR but also on number of RBs assigned per frame. In the new cell selection proposed algorithm, new user calculates the theoretical achievable data rate based on instantaneous SNR and receives Reduction Factor (RF) then calculates the maximum 
effective achievable Data Rate.Formula in (3) stated that according to instantaneous SNR, new user can attain an achievable data rate from table 1 assuming that user is scheduled one per subframe. After calculating number of RBs expected to be assigned to new user, Reduction Factor (RF) due to cell load can be calculated as shown

$$
\mathrm{RF}=\frac{n_{u+1}^{R B}}{N_{s f^{*}} N_{f r}}
$$

Where $n_{u+1}^{R B}$ is obtained from (6) and $N_{s f}$ is number of subframes/frame and $N_{f r}$ is number of frames during prediction interval .It can be noticed that values of RF depends on criteria of mapping SNR values into data rates as in Table 1 whereas values in Table 1 can be achieved if the user is scheduled once every subframe. Figure 1 summarizes the new proposed prediction algorithm.

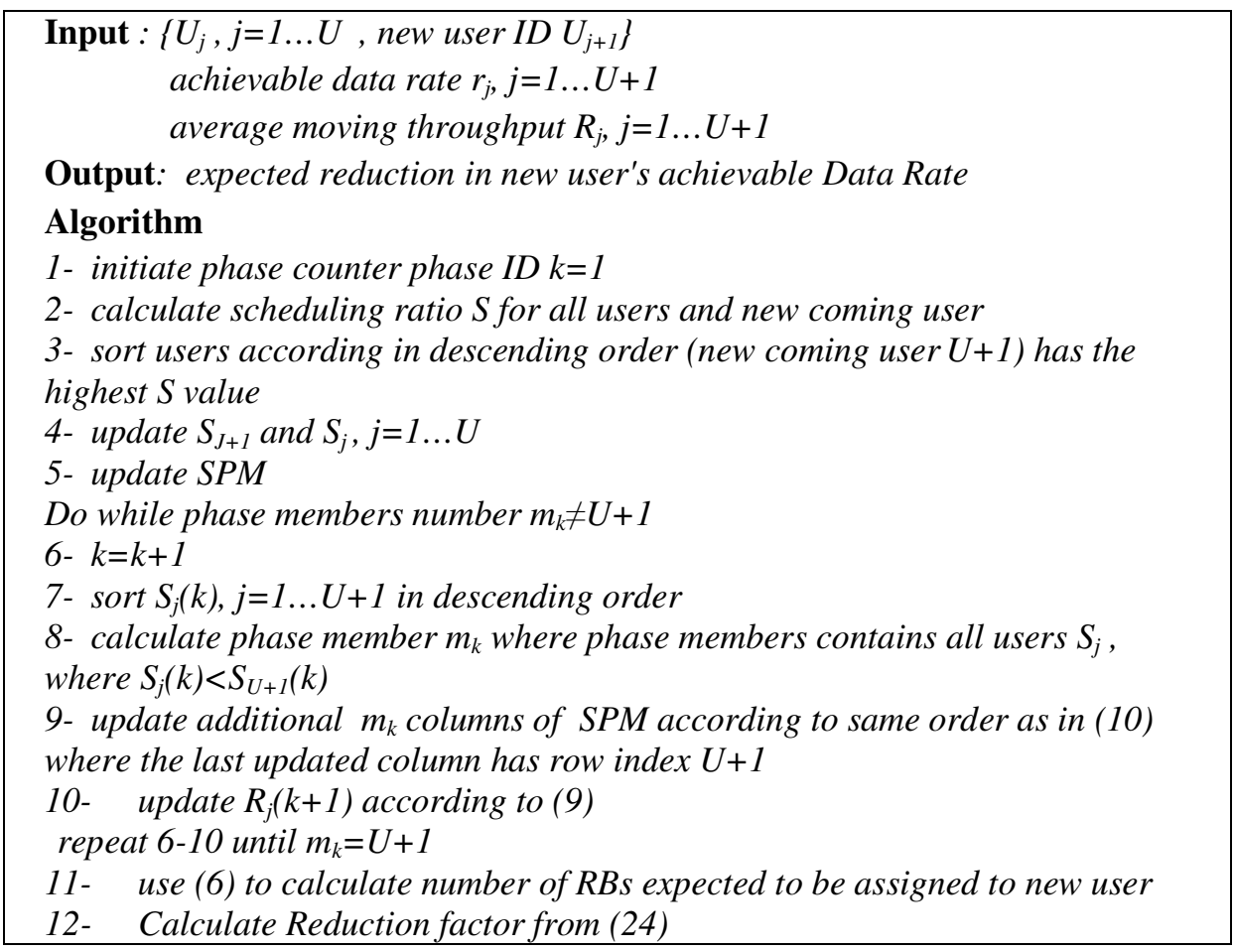

Figure.1: Summary of modelling of PF scheduling algorithm

Hence the effective Data rate of the new user for each of the surrounding cells is given by

$$
\text { effective } D R=R F * r_{u+1}
$$

To verify the accuracy of the prediction algorithm, we perform a simulation to predict the effective data rate of new user using classical prediction algorithm implementing PF criteria after each RB for the same prediction interval as in Fig. 2, we assume here a single cell simulation consisting of several number of users, the value of effective data rate for new user is calculated for all possible values of data rates, the users are assumed to be disturbed uniformly within the cell coverage area. It can be shown that for constant SNR values during the prediction interval, proposed prediction algorithm outputs the same values of effective data rate for new user as a classical prediction algorithm that needs a massive calculation especially with long term prediction. 


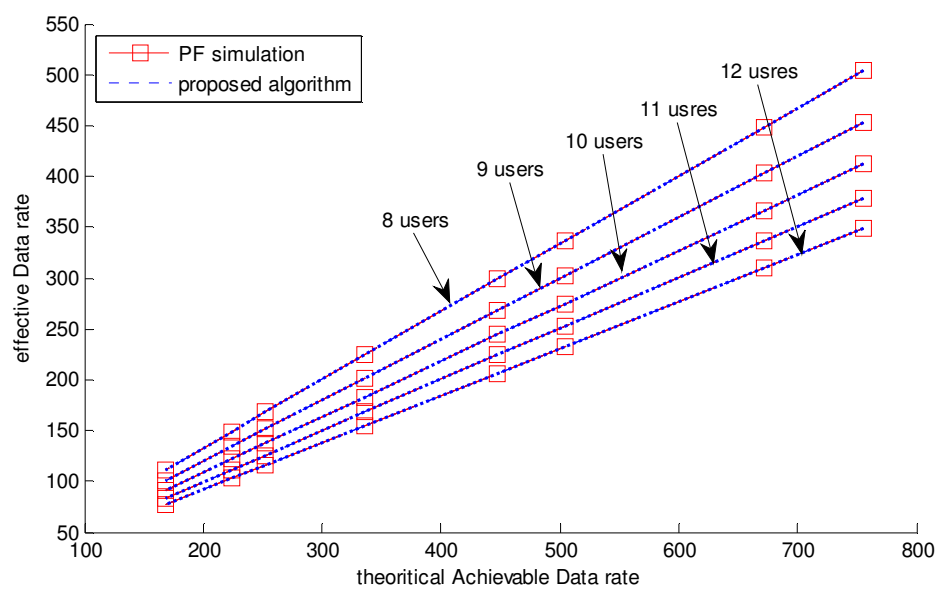

Figure 2. Comparison between classical prediction algorithm and proposed algorithm

\subsection{Throughput analysis}

When studying throughput analysis, we can notice that the total throughput during the simulation time is affected by the new user achievable data rate where the total cell throughput can be increased or decreased as shown in Fig. 3. The total cell throughput for single cell consisting of 8 uniformly distributed users is calculated before and after the new user is being scheduled. It can be shown that the new user can lead to increase or decrease the total cell throughput according to the new users achievable data rate, therefore a new constrain is added to our cell selection criteria to maintain both individual user improved data rate and total cell throughput. The total cell throughput is directly proportional to achievable data rate of the user, so it is preferred that new user's achievable data rate achieves total throughput greater that the total throughput before scheduling new user, the predicted total cell throughput is given by

$$
\text { average cell throughput }=\sum_{i}^{u+1}\left(n_{i}^{R B} * r_{i}\right) /\left(N_{f r} * N_{s f}\right)
$$

The achievable data rate is calculated with assumption that user is served once per subframe i.e. once every $1 \mathrm{~ms}$

Therefore

$$
\text { user throughput }{ }_{i}=\frac{n_{i}^{R B} * r_{i}}{20 n_{f}}
$$

$\therefore$ average cell throughput before sceduling new user $=\sum_{i=1}^{u} \frac{n_{i}^{R B_{*}} r_{i}}{20 n_{f}}$

the following inequality has to be fulfilled to increase total throughput

average cell througput after scheduling new user

$>$ average cell througput before scheduling new user

then

$$
\sum_{i=1}^{u} \frac{n_{i}^{R B} * r_{i}}{20 n_{f}}<\sum_{i=1}^{u+1} \frac{m_{i}^{R B} * r_{i}}{20 n_{f}}
$$

where 
$n^{R B}$ : number of RBs assigned to user $i$ before new user is scheduled

$m^{R B}$ : number of RBs assigned to user $i$ after new user is scheduled

$n_{f}$ :number of frames

number of RBs/subframe $=2$

number of subframes $/$ frame $=10$

For long simulation time i.e. large number of RBs, we need to calculate a lower bound, depending on rule 5 we can neglect the number RBs assigned to user within transient period, number of RBs assigned to user can be approximated to RR performance and equals to

$$
\begin{aligned}
& \frac{\text { total number of } R B s}{\text { number of users }}=\frac{12 * 10 * n_{f}}{u} \\
& \text { where } n_{i}^{R B} \cong \frac{120 * n_{f}}{u} \text { and } m_{i}^{R B} \cong \frac{120 * n_{f}}{u+1}
\end{aligned}
$$

Then

$$
\begin{aligned}
& \sum_{i=1}^{u} \frac{120 n_{f} * r_{i}}{u * n_{f}}<\sum_{i=1}^{u+1} \frac{120 n_{f} * r_{i}}{(u+1) n_{f}} \\
& r_{u+1}>\frac{\sum_{i=1}^{u} r_{i}}{u}
\end{aligned}
$$

Therefore the achievable data rate of the new user must be greater that $\frac{\sum_{i=1}^{u} r_{i}}{u}$ so that the new user scheduling yields to an expected increase in total cell throughput

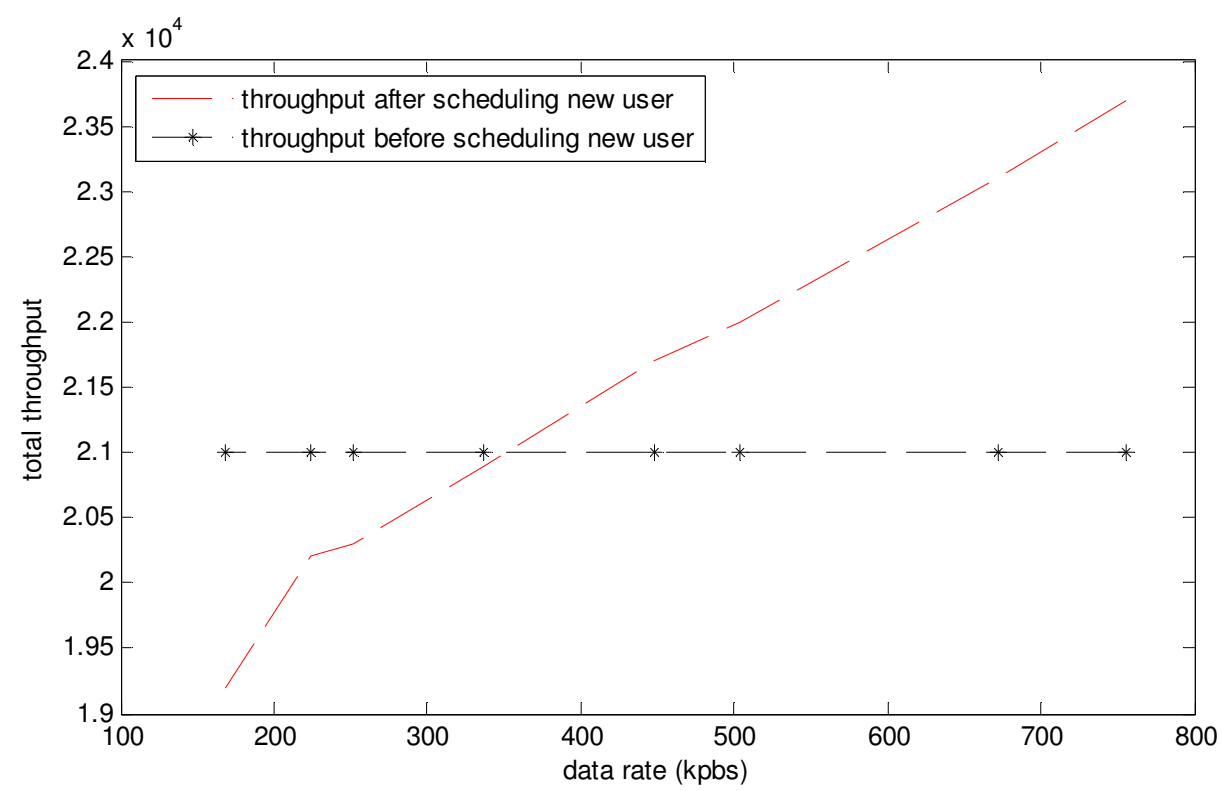

Figure3. Effect of new user's achievable data rate on cell average throughput

\section{PROPOSED CELL SELECTION ALGORITHM}

In this section, we propose two schemes for our new proposed cell selection algorithm, both schemes use prediction algorithm as a main algorithm for calculating the expected number of RBs, however first scheme can need a to be implemented partially in a central node so it can be considered as a hybrid scheme while the second scheme is considered as a standalone algorithm. 
International Journal of Wireless \& Mobile Networks (IJWMN) Vol. 5, No. 6, December 2013

\subsection{Proposed Hybrid Cell Selection algorithm (HCSA)}

The target of the proposed cell selection algorithm is to calculate the reduction in achievable Data rate due to cell load, therefore we need to determine the values of SNR measured by new user with respect to all surrounding cells then simulate the behaviour of user within each surrounding cell to calculate the maximum effective data rate. In the hybrid proposed cell selection algorithm, the procedure is divided between the new user and a central node where the new user measures the value of instantaneous SNR w.r.t all surrounding cells. Through one of surrounding cells, the central node is fed back by the information of each cell load i.e. number of users and their SNR values then the central node runs the proposed prediction algorithm to estimate the effective data rate of the new user for each of the surrounding cells by using equation (25).

The central node sends the ID of the cell achieving maximum effective Data rate to the new user preserving the cell throughput constrain in (32). Therefore the new user transmits instantaneous SNR w.r.t all surrounding cells to a central node via one of surrounding cells, central node calculates the expected reduction in achievable data rate of the new user for each cell then reports the reduction to the new user. The new user selects the cell providing the user with maximum effective achievable data as follows

$$
\text { Cell selected }(c)=\arg \max _{(c)}\left\{R F_{u+1}^{c} * r_{u+1}^{c}\right\} \quad \text { s.t. } r_{u+1}>\frac{\sum_{i=1}^{u} r_{i}}{u}
$$

Proposed cell selection algorithm is summarized in Fig. 2

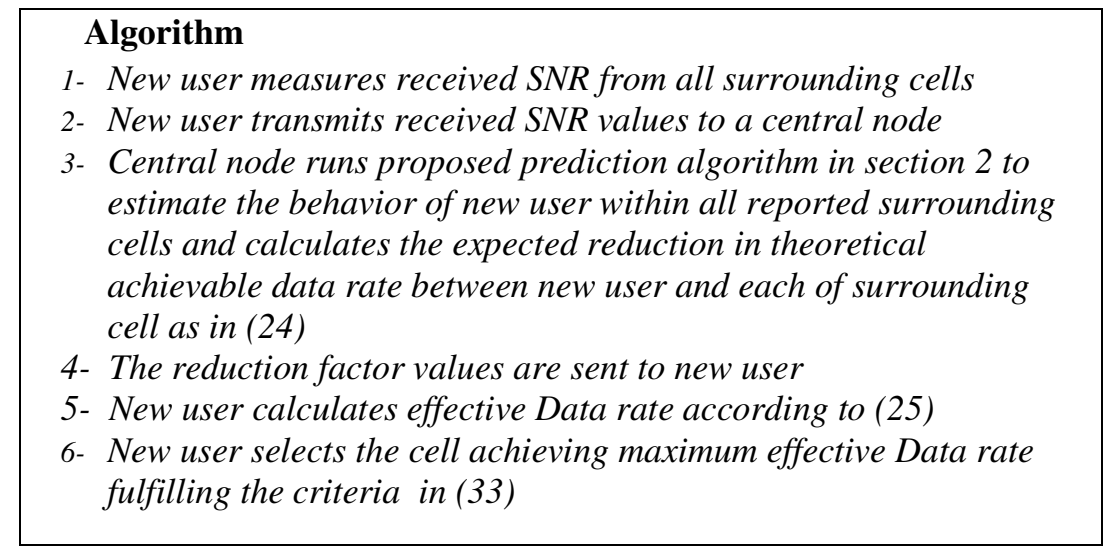

Figure 4. Summary of HCSA.

\subsection{Proposed Standalone Cell Selection Algorithm (SCSA)}

Hybrid cell selection algorithm needs some sort of coordination and signalling overhead between new user and serving cells and also an existence of a central node which will causes an overhead over the signalling links. Considering that users are served according to the maximization of scheduling ratio $S$ and values of average moving throughput $R$ is updated according to (2).

We can apply a constant scaling factor to all values $R_{i}$ such that $R_{i}<<r_{i}$, then the values of $\mathrm{S}$ after substituting (2) in (5) will be almost independent of values of $r$, so each cell can calculate an expected reduction factor regardless the achievable of new user by using an arbitrary value for achievable data rate of new user. Therefore signalling overhead can be omitted whereas each cell calculates its expected reduction factor for new user's achievable data rate then broadcasts it to the new user. New user then calculates effective Data Rate according to measured SNR and received 
reduction factor values without any necessity for processing in central node or data exchange, however the constrain in (31) cannot be fulfilled in standalone algorithm because it needs data exchange between adjacent cells. Fig.3 summarizes the standalone new proposed cell selection algorithm.

\begin{tabular}{|l|}
\hline Algorithm \\
1- Each cell calculates the reduction according as in (24) to its \\
own load by assigning an arbitrary value for new user's \\
achievable Data rate and runs prediction algorithm \\
2- Each cell broadcasts the reduction factor to surrounding users \\
3- new user measures the instantaneous SNR and calculates the \\
achievable Data rate then multiplies it by the broadcasted \\
reduction factor to obtain effective Data rate for all \\
surrounding cells according to (25) \\
4- New user selects the cell achieving maximum effective Data \\
rate applying criteria in (33)with no constrains
\end{tabular}

Figure 5. Summary of SCSA.

\subsection{Simple Prediction Cell Selection Algorithm(SPCSA)}

We propose a simple cell selection algorithm similar to SCSA however the calculations of the reduction factor assume new user will be served in a RR pattern through the entire prediction interval, therefore the reduction factor is calculates as

$R F_{S P C S A}=\frac{R B n o}{U+1}$

Where RBno is thenumber of RBs per TTI, then effective data rate can be calculated using equation (25)

\section{SIMULATION RESULTS}

A multi-cell system level simulation using Matlab for a LTE network is performed in this section, as illustrated in Fig. 6, for a hexagonal cell layout with 7 cells and 3 sectors/cell, each are $120^{\circ}$ apart. Two femtocells are assumed to be randomly located in each sector. Table 2 summarizes simulation parameters. Six cell selection schemes are investigated as shown in Table 3. We first simulate the behaviour of a single user attempting to perform a cell selection over an existing network, the new user is assumed to be located within the coverage area of one of the existing femtocells. Figure 7 plots CDF of the average throughput of the new user. We notice here that the new proposed schemes (scheme 4 and scheme 5) outperform conventional schemes (scheme 1 and scheme 2) by approximately 30\% increase in average user's throughput, however scheme 3 and scheme 6 achieves almost the same performance as proposed schemes(scheme 4 and scheme $5)$. When investigating the rate of femtocell selection, new proposed schemes could have a moderate and acceptable femtocell selection percentage than conventional schemes, where schemes 1 and 2 have a very low femtocell selection rate while scheme 3 have a very high rate which is considered as an advantage due to small coverage area of femtocells and overlaying coverage are between macrocells and femtocells as shown in Table 4. In Figure 8, we investigate the new user's average throughput where the user is assumed to be located in a macro cell coverage area, the proposed schemes (scheme 4 and scheme 5) achieve higher Data rates than conventional schemes (schemes 2 and 3) by $14 \%$ and $6 \%$ respectively while the performance of scheme 1 has almost the same average throughput as proposed schemes, still scheme 6 has the same performance as proposed schemes (schemes 4 and 5), we also notice here that scheme 3 
that shows a good performance in case user is in femtocell coverage while its performance shows a degradation in case user is located in the coverage area of a macrocell .

Figure 9 examines an entire cluster applying addressed cell selection schemes, it can be shown that new proposed schemes achieves about $12 \%$ and $3 \%$ improvement in average cell throughput than scheme 2 which is considered as the standard cell selection algorithm in LTE systems, and scheme 3 respectively. The performance of scheme 1 is almost similar to new proposed schemes (schemes 4 and 5). The previous improvements remain almost unchanged when calculating the average cluster's macrocell throughput as in Fig 10. Despite the similarity of average throughput between proposed schemes and schemes 3 and 1 in case of femtocell selection and macrocell selection respectively, the new proposed schemes achieves better fairness among all cells whereas the variance between number of macrocell users in different cells is minimized in case of new proposed schemes as in Fig. 11 which reduces the necessity of Load Balancing algorithms between adjacent cells. Scheme 4 has the best various among all addressed schemes where it has less variance than scheme 5,6 and 3 by almost $8 \%$ and less than schemes 1 and 2 by almost $22 \%$ which indicates that proposed scheme 4 achieves the best fairness among adjacent cells as users are assumed to be distributed uniformly within the macrocell coverage area.

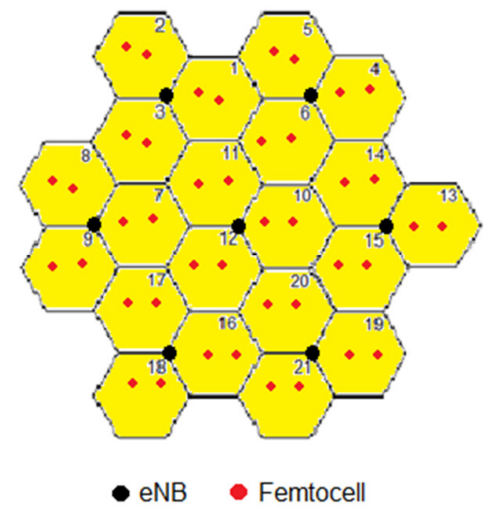

Figure 6. Cell layout.

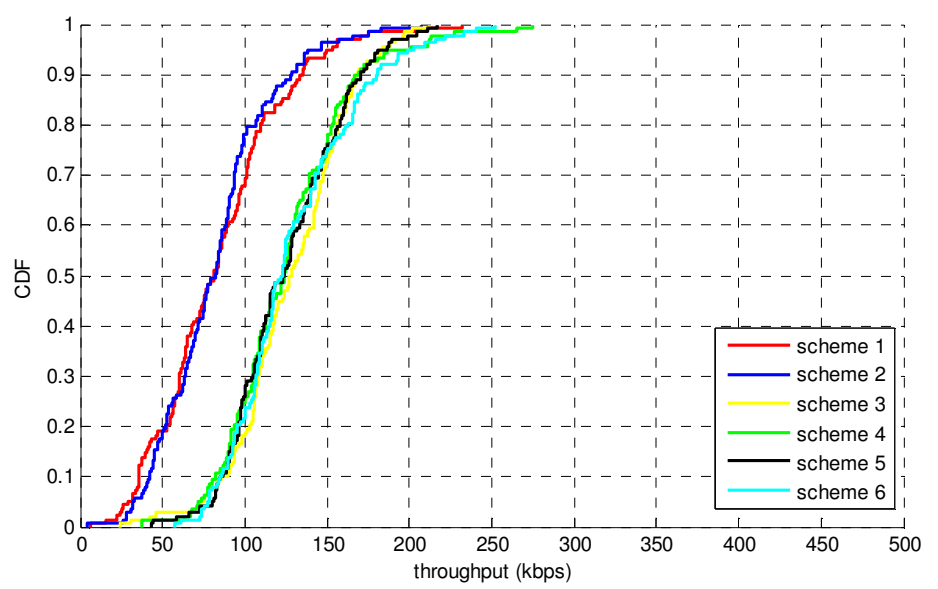

Figure 7. New user's average throughput ( located in femtocell coverage area) 
International Journal of Wireless \& Mobile Networks (IJWMN) Vol. 5, No. 6, December 2013

Table 2. Simulation parameters

\begin{tabular}{|c|c|}
\hline Parameter & Setting \\
\hline System bandwidth & $1.2 \mathrm{MHz}$ \\
\hline Carrier frequency & $2 \mathrm{GHz}$ \\
\hline No. of RBs per half TTI $(0.5 \mathrm{~ms})$ & 6 \\
\hline Cell layout & Hexagonal layout,3 sector per site,resue1 \\
\hline Number of sites & $7(=21$ cell)with wrap-around \\
\hline Macrocell transmit power & $43 \mathrm{dBm}$ \\
\hline Intersite distance & $350 \mathrm{~m}$ \\
\hline Max number of users per Macrocell & 50 \\
\hline Macrocell BS Antenna gain & $14 \mathrm{dBi}$ \\
\hline $\begin{array}{l}\text { Dependent path loss (PL) from } \\
\text { Macrocell BS to Macrocell MS }\end{array}$ & $14.2+37.6 \log _{10}(\mathrm{D}$ in $m)$ \\
\hline $\begin{array}{l}\text { Dependent path loss (PL) from } \\
\text { Macrocell BS to femto MS }\end{array}$ & $\begin{array}{c}\text { 14.2+37.6 } \log _{10} \mathrm{D}+\mathrm{PeL}(\mathrm{D} \text { in } m) \text { PeL: } \\
\text { Penetration Loss }\end{array}$ \\
\hline White noise power density & $-174 \mathrm{dBm} / \mathrm{Hz}$ \\
\hline Shadowing fading (SHL) & Log-normal distribution \\
\hline SHL standard deviation & $8 \mathrm{~dB}$ \\
\hline User velocity & $3 \mathrm{Km} / \mathrm{s}$ \\
\hline Penetration Loss (PeL) & $10 \mathrm{~dB}$ \\
\hline Femtocell Radius & $20 \mathrm{~m}$ \\
\hline No. of femtocells BS per macrocell & 2 \\
\hline $\begin{array}{l}\text { Min. distance between Macrocell and } \\
\text { Femtocell }\end{array}$ & 35 meters \\
\hline $\begin{array}{l}\text { Dependent path loss (PL) from } \\
\text { Femtocell BS to femtocell MS }\end{array}$ & $\begin{array}{c}38.46+20 \log _{10}(\mathrm{D} \text { in } m)+0.7 * \mathrm{~d}[\mathrm{~dB}] \mathrm{d} \\
\text { [m]:distance inside house (assumed to be } 2 \mathrm{~m} \text { ) }\end{array}$ \\
\hline $\begin{array}{l}\text { Dependent path loss (PL) from } \\
\text { Femtocell BS to Macrocell MS }\end{array}$ & $\begin{array}{c}\operatorname{Max}\left(14.2+37.6 \log _{10}(\mathrm{D}), 38.46+20 \log _{10}(\mathrm{D})+\right. \\
0.7 * \mathrm{~d}+\mathrm{PeL})\end{array}$ \\
\hline $\begin{array}{l}\text { Path loss from femtocell BS to } \\
\text { femtocell MS in another femtocell }\end{array}$ & $\begin{array}{c}\operatorname{Max}\left(14.2+37.6 \log _{10}(\mathrm{D}), 38.46+20 \log _{10}(\mathrm{D})+\right. \\
0.7 * \mathrm{~d}+2 * \mathrm{PeL})\end{array}$ \\
\hline $\begin{array}{l}\text { Shadowing fading standard deviation } \\
\text { (indoor environment) }\end{array}$ & $4 \mathrm{~dB}$ \\
\hline Femtocell transmit power & $10 \mathrm{dBm}$ \\
\hline Maximum no. of femtocell users & 10 \\
\hline
\end{tabular}

Table 3. Simulation scenarios

\begin{tabular}{|c|c|}
\hline Scheme no & Scheme type \\
\hline Scheme 1 & Highest SINR \\
\hline Scheme 2 & Highest RSRP \\
\hline Scheme 3 & Minimum path loss \\
\hline Scheme 4 & Proposed HCSA \\
\hline Scheme 5 & Proposed SCSA \\
\hline Scheme 6 & Proposed SPCSA \\
\hline
\end{tabular}


International Journal of Wireless \& Mobile Networks (IJWMN) Vol. 5, No. 6, December 2013

Table 4. Percentage of femtocell selection

\begin{tabular}{|c|c|}
\hline Scheme ID & $\begin{array}{c}\text { Percentage of } \\
\text { femtocell } \\
\text { selection }\end{array}$ \\
\hline Scheme 1 & $21.8 \%$ \\
\hline Scheme 2 & $15 \%$ \\
\hline Scheme 3 & $91.2 \%$ \\
\hline Scheme 4 & $36.4 \%$ \\
\hline Scheme 5 & $38.6 \%$ \\
\hline Scheme 6 & $48.9 \%$ \\
\hline
\end{tabular}

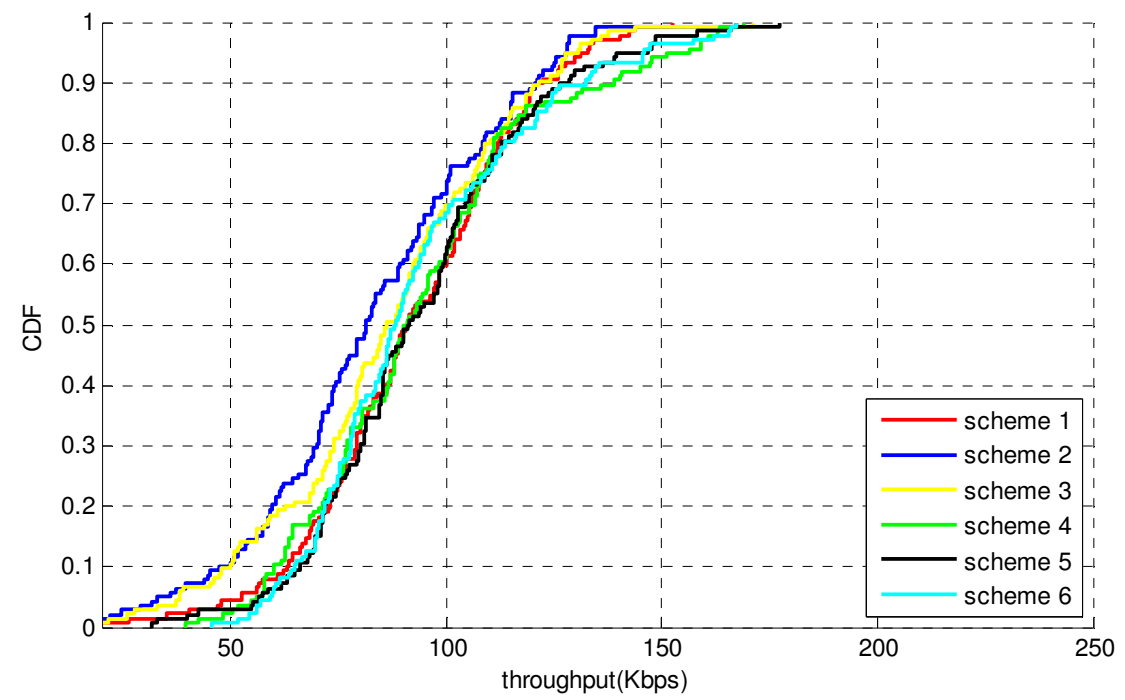

Figure 8 . New user's average throughput (located in macrocell coverage area)

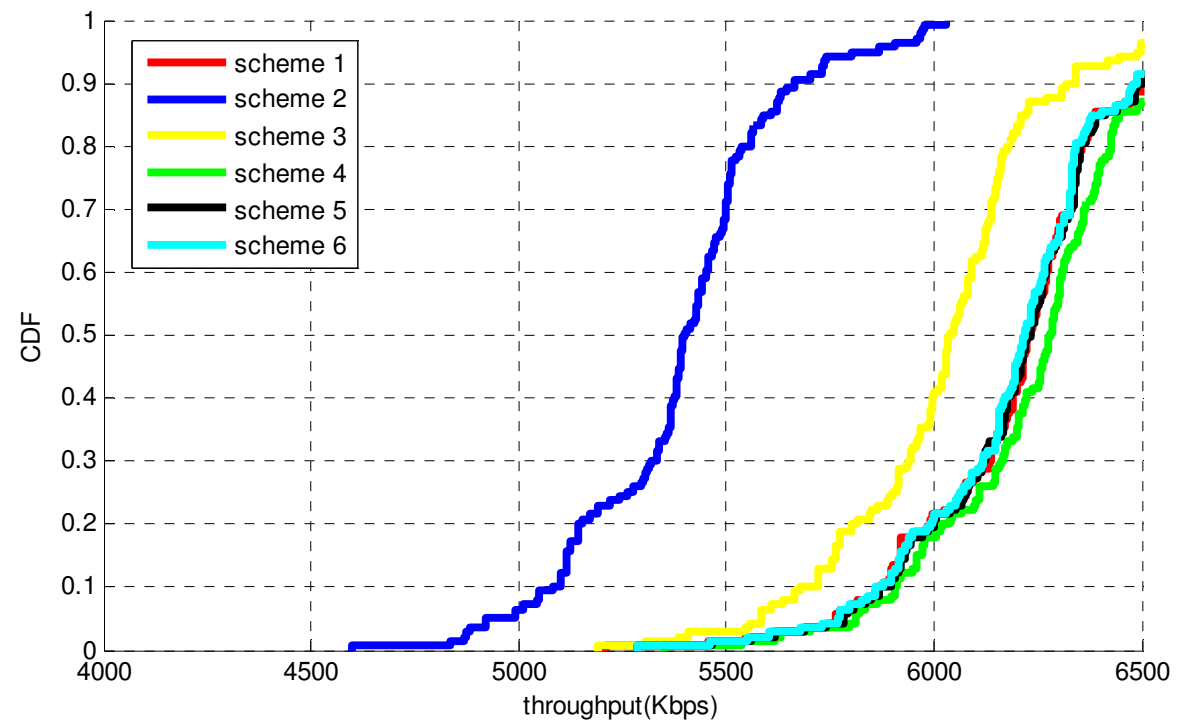


Figure 9. Average cell throughput of addressed cluster

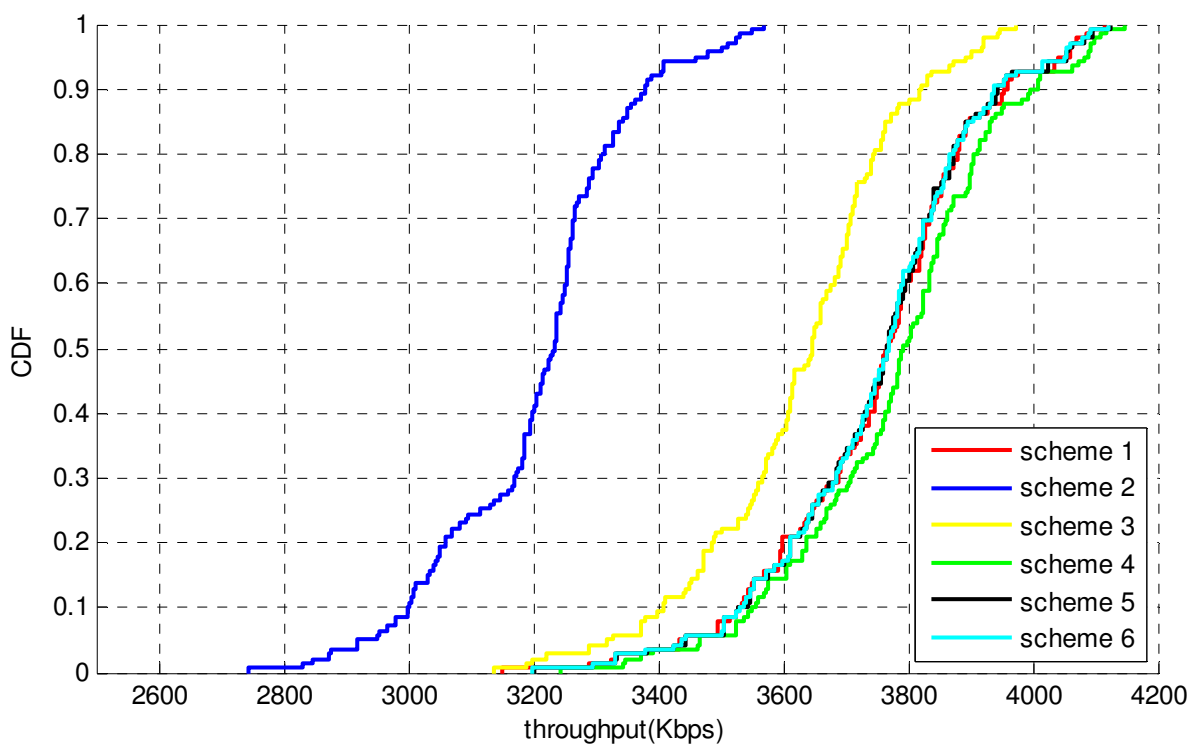

Figure 10. Average macrocell throughput of addressed cluster

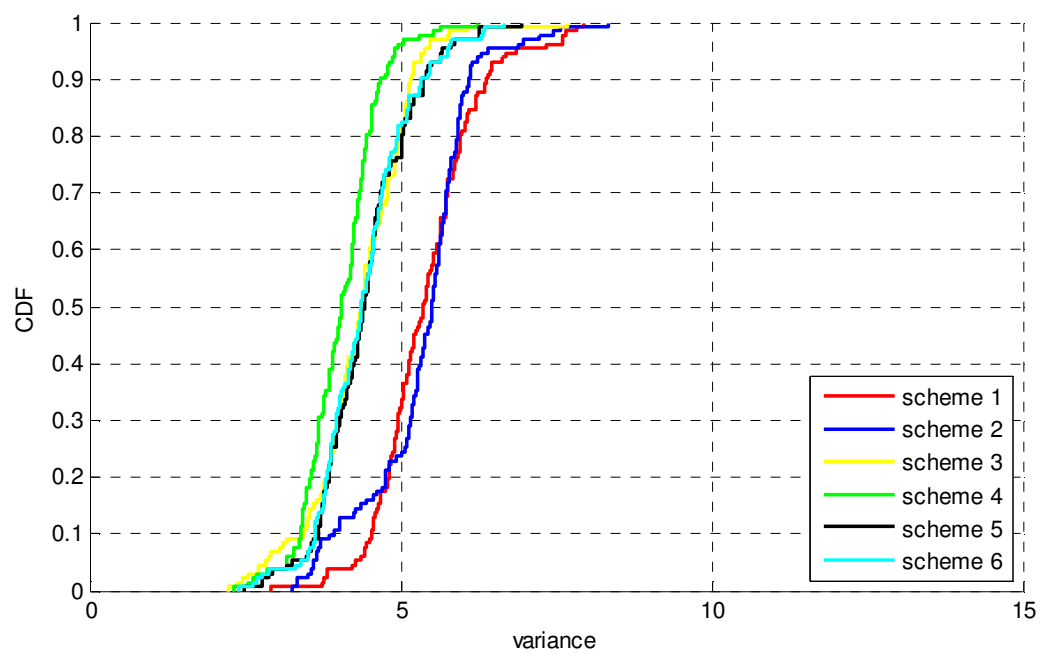

Figure 11. variance between numbers of users per macrocell

\section{CONCLUSION}

In this paper, we studied the cell selection operation in LTE Heterogeneous Networks, firstly we designed a prediction algorithm to estimate the expected number of RBs that will be assigned to a new user for a certain predetermined prediction interval without need to apply PF algorithm after each RB for the entire interval, then a new cell selection algorithm is proposed to calculate the expected reduction in new coming user's achievable data rate. 3 schemes of our new proposed cell selection algorithm have been addressed. The new proposed schemes can be implemented in both hybrid and standalone modes. Simulation results show that the new proposed schemes achieves better values for both user's average Data rate and average cell throughput than conventional 
International Journal of Wireless \& Mobile Networks (IJWMN) Vol. 5, No. 6, December 2013

scheme 2 which is considered as the standard LTE cell selection algorithm, also other conventional cell selection algorithms fail to achieve the best performance in both femtocell and macrocell environment where scheme 3 achieves almost a good performance as proposed schemes in case user is located in a femtocell coverage area while scheme 1 achieves a good performance as proposed schemes if users are located in macrocell coverage area, proposed schemes maintain their outperformed performance in both cases. In addition to improved average throughput, proposed scheme 4 has the best fairness among adjacent cells and thus reduces the need for load balancing algorithms that need a lot of time consuming complex algorithms and signalling overhead.

\section{REFERENCES}

[1] 3GPP, www.3gpp.org.

[2] H. Zhang ; X. Qiu ; L. Meng ; X. Zhang, (2010)"Design of Distributed and Autonomic Load Balancing for Self-Organization LTE ",in IEEE 72nd VTC 2010-Fall ,pp. 1 - 5.

[3] H. Wang ; L. Ding ; P. Wu ; Z. Pan ; N. Liu ; X. You (2010) "Dynamic load balancing in 3GPP LTE multi-cell networks with heterogeneous services" 5th International ICST Conference on Communications and Networking in China (CHINACOM), pp. 1 - 5.

[4] Siomina, I. ; Di Yuan, (2012)"Load balancing in heterogeneous LTE: Range optimization via cell offset and load-coupling characterization", in IEEE International Conference on Communications (ICC) ,pp. 1357 - 1361.

[5] Keon-Wook Lee, Jae-Yun Ko, Yong-Hwan Lee,(2006) "Fast Cell Site Selection with Interference Avoidance in Packet Based OFDM Cellular Systems", IEEE GLOBECOM '06,pp.1-5

[6] A. Sang, X. Wang, M. Madihian, and R. Gitlin, (2004)“Coordinated load balancing, handoff/cell-site selection, and scheduling in multi-cell packet data systems," in Proc. ACM Mobicom, pp. 302-314.

[7] T.Qu; D. Xiao; D.Yang,( 2010 ), "A novel cell selection method in heterogeneous LTE-advanced systems " in IEEE 3rd International Conference on Broadband Network and Multimedia Technology (IC-BNMT), Beijing, pp. 510 - 513.

[8] J. Sangiamwong, Y. Saito, N. Miki, T. Abe, S. Nagata, and Y.Okumura, (2011) " Investigation on Cell Selection Methods Associated with Inter-cell Interference Coordination in Heterogeneous Networks for LTE-Advanced Downlink" in IEEE 11th European Wireless Conference, Vienna, pp. $1-6$.

[9] Simsek, M.; Hanguang Wu; Bo Zhao; Akbudak, T.; Czylwik, A., (2011), " Performance of Different Cell Selection Modes in 3GPP-LTE Macro-/Femtocell Scenarios" in IEEE Wireless Advanced (WiAd), pp. 126-131.

[10] T. Qu ; D. Xiao ; D. Yang ; W. Jin ; Y. He, (2010)," Cell selection analysis in outdoor heterogeneous networks ", 3rd International Conference on Advanced Computer Theory and Engineering (ICACTE), pp.V5-554- 557.

[11] RI-083813, "Range expansion for efficient support of heterogeneous networks," Qualcomm Europe.

[12] J. Wang; J. Liu; D. Wang; J. Pang; G. Shen, (2011)," Optimized Fairness Cell Selection for 3GPP LTE-A Macro-Pico HetNets", IEEE Vehicular Technology Conference (VTC Fall), pp. 1-5.

[13] Amzallag, D. Bar-Yehuda, R. Raz, D. Scalosub, (2013),"Cell Selection in 4G Cellular Networks", IEEE Transactions on Mobile Computing, vol. 12, no.7, pp. 1443 - 1455.

[14] A. Jalali, R. Padovani, and R. Pankaj, (2000), "Data Throughput of CDMAHDR a High EfficiencyHigh Data Rate Personal Communication Wireless System," in IEEE 51st Vehicular Technology Conference Proceedings, Tokyo, pp. 1854-1858. 
International Journal of Wireless \& Mobile Networks (IJWMN) Vol. 5, No. 6, December 2013

[15] C. Westphal,( 2004), "Monitoring proportional fairness in cdma2000 high data rate networks," in Proceedings of Globecom, vol. 6, pp. 3866-3871.

[16] B. B. Chen and M. C. Chan,( 2006), "Proportional Fairness for Overlapping Cells in Wireless Networks," VTC 2006-Fall, Montreal, Canada, 25-28 September, pp. 1-5.

[17] E. Liu , K. K. Leung, (2008), "Proportional Fair Scheduling: Analytical Insight under Rayleigh Fading Environment", IEEE WCNC ,pp1883-1888.

[18] J. G. Choi and S. Bahk., (2007)," Cell-Throughput Analysis of the Proportional Fair Scheduler in the Single-Cell Environment", IEEE Transactions on Vehicular Technology, , Vol. 56, No. 2, pp. $766-$ 778 .

[19] X. Qiu and K. Chawla, (1999), "On the Performance of Adaptive Modulation in Cellular Systems," in IEEE Transactions on Communications. vol. 47, 1999, pp. 884-895.

[20] Ramli, H.A.M.; Basukala, R.; Sandrasegaran, K.; Patachaianand, R., (2009), "Performance of Well Known Packet Scheduling Algorithms in the Downlink 3GPP LTE System", IEEE 9th Malaysia International Conference on Communications (MICC) , pp. 815-820. 